PROCEEDINGS OF THE

AMERICAN MATHEMATICAL SOCIETY

Volume 125, Number 1, January 1997, Pages 145-151

S 0002-9939(97)03534-X

\title{
COMPACT COMPOSITION OPERATORS ON THE NEVANLINNA CLASS
}

\author{
JUN SOO CHOA AND HONG OH KIM
}

(Communicated by Theodore W. Gamelin)

\begin{abstract}
In this paper we prove that the composition operator induced by a holomorphic self-map of the unit disc is compact on the Nevanlinna class if and only if it is compact on the Hardy space $H^{2}$.
\end{abstract}

\section{INTRODUCTION}

Let $\varphi$ be a holomorphic self-map of the unit disc $\mathbb{D}$ in the complex plane. Then the equation $C_{\varphi}(f)=f \circ \varphi$ defines a composition operator $C_{\varphi}$ on the space of holomorphic functions in $\mathbb{D}$. As is well known, each $C_{\varphi}$ is a bounded linear operator on the Hardy space $H^{p}$ [Nor, Sch]. It is also known that if $C_{\varphi}$ is compact on one of the spaces $H^{p}$, then it is compact on all of them $(0<p<\infty)$ [ST]. So this result leads us to ask whether the compactness of $C_{\varphi}$ on the Nevanlinna class implies its compactness on the Hardy space $H^{p}$, and conversely. The purpose of this present note is to give an affirmative answer to this question. More precisely we prove:

A composition operator $C_{\varphi}$ is compact on the

Nevanlinna class if and only if it is compact on $H^{2}$.

The composition operator $C_{\varphi}$ as an operator on the Hardy space has been studied by many authors [Cow, Mac, MS, Nor, Sch, Sh2, ST]. Complete characterizations of $\varphi$ for which $C_{\varphi}$ is compact on $H^{2}$ have been given in [Sh1, Sh2, Mac]. However, as far as we know, the operator $C_{\varphi}$ as an operator on the Nevanlinna class was first studied by Masri in his thesis [Mas], where he obtained several necessary conditions and sufficient conditions on $\varphi$ for the operator $C_{\varphi}$ to be compact on the Nevanlinna class. The conditions are in the same spirit as conditions developed in [ST] for studying the compactness of $C_{\varphi}$ on the Hardy space. Our criterion is, however, a complete characterization of $\varphi$ for which $C_{\varphi}$ is compact on the Nevanlinna class. The result of this note relies on Shapiro's Nevanlinna counting function criterion [Sh2] and MacCluer's Carleson-measure criterion [Mac] for the compactness of $C_{\varphi}$ on the Hardy space.

Received by the editors May 2, 1995 and, in revised form, June 30, 1995.

1991 Mathematics Subject Classification. Primary 47B05, 47B38; Secondary 30D55.

Key words and phrases. Compact composition operator, Nevanlinna counting function, Carleson measure.

Supported in part by TGRC, and Korean ministry of Education, BSRI-95-1420.

(C) 1997 American Mathematical Society 


\section{Preliminaries}

2.1. Nevanlinna class. A holomorphic function $f(z)$ in the unit disc $\mathbb{D}$ is said to belong to the Nevanlinna class $N$ if

$$
\lim _{r \nearrow 1} \int_{0}^{2 \pi} \log ^{+}\left|f\left(r e^{i \theta}\right)\right| \frac{d \theta}{2 \pi}<\infty .
$$

Obviously, the inequalities

$$
2 \log ^{+} x \leq \log \left(1+x^{2}\right) \leq 1+2 \log ^{+} x, \quad x \geq 0,
$$

imply that

$$
f \in N \quad \text { if and only if } \quad\|f\|_{N}:=\lim _{r \nearrow 1} \int_{0}^{2 \pi} \log \left(1+\left|f\left(r e^{i \theta}\right)\right|^{2}\right) \frac{d \theta}{2 \pi}<\infty
$$

for $f$ holomorphic in $\mathbb{D}$. For $f, g \in N$, we define $d(f, g)=\|f-g\|_{N}$, then $d$ is a translation invariant metric on $N$ and $(N, d)$ becomes a complete metric space [SS]. For other properties of $(N, d)$ see [Du, Ga].

2.2. Composition operators. In this paper, the symbol $\varphi$ always denotes a holomorphic self-map of $\mathbb{D}$. Each such map $\varphi$ induces a linear composition operator on the space of holomorphic functions on $\mathbb{D}$ by means of the equation $C_{\varphi}(f)=f \circ \varphi$ $(f$ is holomorphic in $\mathbb{D})$. It follows from the Littlewood subordination principle [Du] (or from the Harnack inequality) that each $C_{\varphi}$ is bounded on the Nevanlinna class $N$. As in [Mas], we say that the operator $C_{\varphi}$ is compact on the class $N$ if the closure of the image, under $C_{\varphi}$, of each bounded set is compact. We recall that a subset $E$ of $N$ is bounded if there exists a finite constant $M$ such that $d(f, g) \leq M$ for all $f, g \in E$.

\section{Compactness of $C_{\varphi}$ on the Nevanlinna Class}

We proceed to present the main result of this paper. As noted in the introduction, what we want to prove is:

Theorem. Suppose $\varphi$ is a holomorphic self-map of $\mathbb{D}$. Then $C_{\varphi}$ is compact on $N$ if and only if it is compact on $H^{2}$.

In order to prove this theorem, we need the next several lemmas.

The first is the following Littlewood and Paley-type identity. The proof below, which is based on Green's formula, can be obtained by a slight modification of that of [Ga, Lemma 3.1]. But it is included for the reader's convenience. Recall that Green's formula states that if $u, v \in C^{2}(\bar{\Omega})(\Omega$ is an open set in the plane with smooth boundary), then

$$
\iint_{\Omega}(u \Delta v-v \Delta u) d x d y=\int_{\partial \Omega}\left(u \frac{\partial v}{\partial n}-v \frac{\partial u}{\partial n}\right) d s
$$

where $\Delta$ is the Laplacian, $\partial / \partial n$ is differentiation in the outward normal direction, and $d s$ is arc length on $\partial \Omega$. In the rest of this paper, $d A$ denotes the normalized Lebesgue area measure on $\mathbb{D}$.

Lemma 1. Suppose $f \in N$. Then

$$
\|f\|_{N}=\log \left(1+|f(0)|^{2}\right)+2 \iint_{\mathbb{D}} \frac{\left|f^{\prime}(z)\right|^{2}}{\left(1+|f(z)|^{2}\right)^{2}} \log \frac{1}{|z|} d A(z)
$$

where, as always "II $\|_{N}$ " denotes the Nevanlinna quasi-norm as defined in Section 2. 
Proof. Let $0<\varepsilon<r<1$. Apply Green's formula (1) with $u(z)=\log (r /|z|)$, $v(z)=\log \left(1+|f(z)|^{2}\right)$, and $\Omega=\{z \in \mathbb{D}: \varepsilon<|z|<r\}$. Since $\Delta u=0$ on $\mathbb{D} \backslash\{0\}$, the result (after rearrangement) is

$$
\begin{gathered}
\iint_{\varepsilon<|z|<r} \Delta \log \left(1+|f(z)|^{2}\right) \log \frac{r}{|z|} d x d y+\log \frac{\varepsilon}{r} \int_{|z|=\varepsilon} \frac{\partial}{\partial n}\left(\log \left(1+|f(z)|^{2}\right)\right) d s \\
=\int_{|z|=r} \frac{\log \left(1+|f(z)|^{2}\right)}{r} d s-\int_{|z|=\varepsilon} \frac{\log \left(1+|f(z)|^{2}\right)}{\varepsilon} d s .
\end{gathered}
$$

Since $\frac{\partial}{\partial n}\left(\log \left(1+|f(z)|^{2}\right)\right)$ is bounded near 0 , the second term of (3) tends to 0 as $\varepsilon \searrow 0$. Note that, on $|z|<r$,

$$
\Delta\left(\log \left(1+|f(z)|^{2}\right)\right)=\frac{4\left|f^{\prime}\right|^{2}}{\left(1+|f(z)|^{2}\right)^{2}}
$$

and therefore, taking the limit $\varepsilon \searrow 0$ in (3) (recall that $\log \frac{1}{|z|}$ is integrable near 0 ), we get

$$
\begin{aligned}
& 4 \iint_{|z|<r} \frac{\left|f^{\prime}(z)\right|^{2}}{\left(1+|f(z)|^{2}\right)^{2}} \log \frac{r}{|z|} d x d y \\
& \quad=\int_{0}^{2 \pi} \log \left(1+\left|f\left(r e^{i \theta}\right)\right|^{2}\right) d \theta-2 \pi \log \left(1+|f(0)|^{2}\right) .
\end{aligned}
$$

Observe that $\log \frac{r}{|z|} \geq 0$ on $|z|<r$ and $\log \frac{r}{|z|} \nearrow \log \frac{1}{|z|}$ as $r \nearrow 1$. Thus by the monotone convergence theorem we have

$$
\begin{aligned}
& 2 \iint_{\mathbb{D}} \frac{\left|f^{\prime}(z)\right|^{2}}{\left(1+|f(z)|^{2}\right)^{2}} \log \frac{1}{|z|} d A(z) \\
& \quad=\lim _{r \nearrow 1} \int_{0}^{2 \pi} \log \left(1+\left|f\left(r e^{i \theta}\right)\right|^{2}\right) \frac{d \theta}{2 \pi}-\log \left(1+|f(0)|^{2}\right),
\end{aligned}
$$

which proves the lemma.

The next is a special case of Stanton's formula [Sh1, Stn].

Lemma 2. Suppose $f \in N$ and $\varphi$ is a holomorphic self-map of $\mathbb{D}$. Then

$$
\iint_{\mathbb{D}} \frac{\left|f^{\prime}(\varphi)\right|^{2}}{\left(1+|f(\varphi)|^{2}\right)^{2}} \|\left.\varphi^{\prime}\right|^{2} \log \frac{1}{|z|} d A(z)=\iint_{\mathbb{D}} \frac{\left|f^{\prime}(w)\right|^{2}}{\left(1+|f(w)|^{2}\right)^{2}} N_{\varphi}(w) d A(w),
$$

where $N_{\varphi}(w)$ is the (usual) Nevanlinna counting function defined by

$$
N_{\varphi}(w)= \begin{cases}\sum_{z \in \varphi^{-1}(w)} \log \frac{1}{|z|} & \text { if } w \in \varphi(\mathbb{D}), \\ 0 & \text { if } w \notin \varphi(\mathbb{D}) .\end{cases}
$$

The following result is an immediate consequence of the two formulas (2) and (7). 
Lemma 3. Under the same hypothesis as in Lemma 2, we have

$$
\left\|C_{\varphi} f\right\|_{N}=\log \left(1+|f(\varphi(0))|^{2}\right)+2 \iint_{\mathbb{D}} \frac{\left|f^{\prime}(w)\right|^{2}}{\left(1+|f(w)|^{2}\right)^{2}} N_{\varphi}(w) d A(w) .
$$

The above lemma suggests that the Nevanlinna counting function is closely related to composition operators on the Nevanlinna class.

The following is a characterization of compactness of $C_{\varphi}$ expressed in terms of sequential convergence, which is taken from [Mas, Theorem 2.4.2].

Lemma 4. Let $\varphi$ be a holomorphic self-map of $\mathbb{D}$. Then $C_{\varphi}$ is a compact operator on $N$ if and only if for every sequence $\left\{f_{n}\right\}$ which is bounded in $N$ and converges to zero uniformly on compact subsets of $\mathbb{D}$, we have $\left\|f_{n} \circ \varphi\right\|_{N} \rightarrow 0$.

The next criteria of compactness of $C_{\varphi}$, which are due to Shapiro [Sh2] and MacCluer [Mac], are useful in the proof of the main result of this note. In what follows, we say that a positive measure $\mu$ on $\overline{\mathbb{D}}$ is a little Carleson measure if

$$
\lim _{\delta \rightarrow 0} \frac{\mu\left(S_{\delta}(\zeta)\right)}{\delta}=0 \quad \text { uniformly in } \zeta \in \partial \mathbb{D}
$$

where $S_{\delta}(\zeta)=\left\{r e^{i t} \in \overline{\mathbb{D}}: 1-\delta<r \leq 1\right.$ and $\left.\left|e^{i t}-\zeta\right|<\delta\right\}$.

Lemma 5. For $\varphi$ a holomorphic self-map of $\mathbb{D}$, the following conditions are equivalent:

(a) $C_{\varphi}$ is compact on $H^{2}$.

(b) $\lim _{|w| \nearrow 1} \frac{N_{\varphi}(w)}{1-|w|}=0$.

(c) The pull-back measure $\mu_{\varphi}$ defined by $\mu_{\varphi}=\sigma \circ \varphi^{-1}$ is a little Carleson measure on $\overline{\mathbb{D}}$; here $\sigma=d \theta / 2 \pi$.

We are now ready to prove the main result of this paper.

Proof of the Theorem. First we assume that $C_{\varphi}$ is compact on $H^{2}$ and will show that $C_{\varphi}$ is compact on $N$. The argument to prove this part here is very similar to that of $\S 10.5$ in [Sh1]. For this, fix a sequence $\left\{f_{n}\right\}$ that is bounded, by a finite constant $M$, in the class $N$ and converges to zero uniformly on compact subsets of $\mathbb{D}$. By Lemma 4 , it is enough to show that $\left\|f_{n} \circ \varphi\right\|_{N} \rightarrow 0$.

Let $\varepsilon>0$ be given. Then it follows from Lemma 5 that we can choose $0<r<1$ such that

$$
N_{\varphi}(w)<\varepsilon \log \frac{1}{|w|} \quad \text { whenever } r \leq|w|<1
$$

Since $f_{n} \rightarrow 0$ uniformly on compact subsets of $\mathbb{D}$, so is $f_{n}^{\prime}$. Thus we can choose $n_{\varepsilon}$ so that

$$
\left|f_{n}\right| \text { and }\left|f_{n}^{\prime}\right|<\sqrt{\varepsilon}
$$


on $r \mathbb{D} \cup\{\varphi(0)\}$ whenever $n>n_{\varepsilon}$. Hence for each such $n$ we have from formula (8)

$$
\begin{aligned}
\left\|f_{n} \circ \varphi\right\|_{N}= & \log \left(1+\left|f_{n}(\varphi(0))\right|^{2}\right) \\
& +2\left[\iint_{r \mathbb{D}}+\iint_{\mathbb{D} \backslash r \mathbb{D}} \frac{\left|f_{n}^{\prime}(w)\right|^{2}}{\left(1+\left|f_{n}(w)\right|^{2}\right)^{2}} N_{\varphi}(w) d A(w)\right] \\
\leq & \log (1+\varepsilon)+2 \varepsilon \iint_{r \mathbb{D}} N_{\varphi}(w) d A(w) \\
& +2 \varepsilon \iint_{\mathbb{D} \backslash r \mathbb{D}} \frac{\left|f_{n}^{\prime}(w)\right|^{2}}{\left(1+\left|f_{n}(w)\right|^{2}\right)^{2}} \log \frac{1}{|w|} d A(w) \\
\leq & \varepsilon+2 \varepsilon \iint_{\mathbb{D}} N_{\varphi}(w) d A(w)+2 \varepsilon \iint_{\mathbb{D}} \frac{\left|f_{n}^{\prime}(w)\right|^{2}}{\left(1+\left|f_{n}(w)\right|^{2}\right)^{2}} \log \frac{1}{|w|} d A(w) \\
\leq & \varepsilon+\varepsilon\left(\|\varphi\|_{N}-\log \left(1+|\varphi(0)|^{2}\right)\right)+\varepsilon\left(\left\|f_{n}\right\|_{N}-\log \left(1+\left|f_{n}(0)\right|^{2}\right)\right) \\
\leq & \varepsilon+\varepsilon\|\varphi\|_{N}+\varepsilon\left\|f_{n}\right\|_{N} \\
\leq & \varepsilon+\varepsilon+\varepsilon M,
\end{aligned}
$$

where in the third-to-last line we have used formula (8) with $f(z)=z$ and Lemma 1 , and in the last line we used the fact that $\left\|f_{n}\right\|_{N} \leq M$ for each $n$. Thus $\left\|f_{n} \circ \varphi\right\|_{N} \rightarrow$ 0 , which establishes the compactness on $N$.

For the converse direction, we now assume $C_{\varphi}$ is compact on $N$. Because of Lemma 5, we only need to verify that the pull-back measure $\mu_{\varphi}=\sigma \circ \varphi^{-1}$ is a little Carleson on $\overline{\mathbb{D}}$.

To prove this, we let $a=(1-\delta) \zeta$ where $\zeta \in \partial \mathbb{D}$ and $0<\delta<1$ and define

$$
f_{a}(z)=(1-|a|) \exp \left(\frac{1+\bar{a} z}{1-\bar{a} z}\right) .
$$

Then $f_{a} \rightarrow 0$ uniformly on compact subsets of $\mathbb{D}$ as $|a| \nearrow 1$. Moreover, a simple calculation, together with the trivial inequality

$$
\log ^{+} x y \leq \log ^{+} x+\log ^{+} y \text { for } x, y \geq 0,
$$

gives

$$
\begin{aligned}
\left\|f_{a}\right\|_{N} & =\lim _{r \nearrow 1} \int_{0}^{2 \pi} \log \left(1+\left|f_{a}\left(r e^{i \theta}\right)\right|^{2}\right) \frac{d \theta}{2 \pi} \\
& \leq 1+2 \lim _{r \nearrow 1} \int_{0}^{2 \pi} \log ^{+}\left|f_{a}\left(r e^{i \theta}\right)\right| \frac{d \theta}{2 \pi} \\
& =1+2 \lim _{r \nearrow 1} \int_{0}^{2 \pi} \log ^{+}\left[(1-|a|) \exp \left(\frac{1-|a|^{2} r^{2}}{\left|1-\bar{a} r e^{i \theta}\right|^{2}}\right)\right] \frac{d \theta}{d \pi} \\
& \leq 1+2 \lim _{r \nearrow 1} \int_{0}^{2 \pi} \frac{1-|a|^{2} r^{2}}{\left|1-\bar{a} r e^{i \theta}\right|^{2}} \frac{d \theta}{2 \pi}=3 .
\end{aligned}
$$

It now follows from the compactness of $C_{\varphi}$ on $N$ and Lemma 4 that

$$
\lim _{|a| \nearrow 1}\left\|f_{a} \circ \varphi\right\|_{N}=0 .
$$

On the other hand, if $z \in S_{\delta}(\zeta)$, then

$$
\frac{1-|a|^{2}|z|^{2}}{|1-\bar{a} z|^{2}} \geq \frac{1-|a|^{2}}{|1-\bar{a} z|^{2}} \geq \frac{1}{5 \delta}
$$


so that

$$
\begin{aligned}
\log ^{+}\left|f_{a}(z)\right| & \geq \log ^{+}\left[(1-|a|) \exp \left(\frac{1-|a|^{2}|z|^{2}}{|1-\bar{a} z|^{2}}\right)\right] \\
& \geq \log ^{+} \delta \exp \left(\frac{1}{5 \delta}\right) .
\end{aligned}
$$

Hence, for all $\zeta \in \partial \mathbb{D}$ and $0<\delta<1$,

$$
\begin{aligned}
& \left(\log ^{+} \delta \exp \left(\frac{1}{5 \delta}\right)\right) \mu_{\varphi}\left(S_{\delta}(\zeta)\right) \leq \iint_{S_{\delta}(\zeta)} \log ^{+}\left|f_{a}(z)\right| d \mu_{\varphi}(z) \\
& \quad \leq \iint_{S_{\delta}(\zeta)} \log \left(1+\left|f_{a}(z)\right|^{2}\right) d \mu_{\varphi}(z) \leq \iint_{\overline{\mathbb{D}}} \log \left(1+\left|f_{a}(z)\right|^{2}\right) d \mu_{\varphi}(z) \\
& \quad \leq \lim _{r \nearrow 1} \int_{0}^{2 \pi} \log \left(1+\left|f_{a} \circ \varphi\left(r e^{i \theta}\right)\right|^{2}\right) \frac{d \theta}{2 \pi}=\left\|f_{a} \circ \varphi\right\|_{N},
\end{aligned}
$$

where the last inequality follows from Fatou's lemma. As we saw above, the compactness of $C_{\varphi}$ on $N$ forces $\left\|f_{a} \circ \varphi\right\|_{N}$ to zero as $|a| \nearrow 1$, which implies

$$
\lim _{\delta \rightarrow 0}\left(\log ^{+} \delta \exp \left(\frac{1}{5 \delta}\right)\right) \mu_{\varphi}\left(S_{\delta}(\zeta)\right)=0
$$

uniformly in $\zeta \in \partial \mathbb{D}$. Therefore the conclusion follows since

$$
\lim _{\delta \rightarrow 0} \delta\left(\log ^{+} \delta \exp \left(\frac{1}{5 \delta}\right)\right)=\lim _{t \rightarrow \infty} \frac{\frac{t}{5}-\log t}{t}=\frac{1}{5} .
$$

\section{ACKNOWLEDGEMENT}

The first author wishes to extend his sincere gratitude to Professor Patrick Ahern for the valuable discussions during his visit to the University of Wisconsin where this work was initiated.

\section{REFERENCES}

[Cow] C. C. Cowen, Composition operators on $H^{2}$, J. Operator Th. 9 (1983), 77-106. MR 84d:47038

[Du] P. L. Duren, Theory of $H^{p}$ spaces, Academic Press, 1970. MR 42:3552

[Ga] J. B. Garnett, Bounded analytic functions, Academic Press, 1981. MR 83g:30037

[Mac] B. D. MacCluer, Compact composition operators on $H^{p}\left(B_{N}\right)$, Michigan Math. J. 32 (1985), 237-248. MR 86g:47037

[Mas] M. I. Masri, Compact composition operators on the Nevanlinna and Smirnov classes, Thesis, University of North Carolina, Chapel Hill, 1985.

[MS] B. D. MacCluer and J. H. Shapiro, Angular derivatives and compact composition operators on the Hardy and Bergman spaces, Canadian J. Math. 38 (1986), 878-906. MR 87h:47048

[Nor] E. Nordgren, Composition operators, Canadian J. Math. 20 (1968), 442-449. MR 36:6961

[Sch] H. J. Schwartz, Composition operators on $H^{p}$, Thesis, University of Toledo, 1969.

[Sh1] J. H. Shapiro, Composition operators and classical function theory, Springer-Verlag, 1993. MR 94k:47049

[Sh2] _ The essential norm of a composition operator, Annals of Math. 125 (1987), 375404. MR 88c: 47058

[SS] J. H. Shapiro and A. L. Shields, Unusual topological properties of the Nevanlinna class, Amer. J. of Math. 97(4), 915-936. 
[ST] J. H. Shapiro and P. D. Taylor, Compact, nuclear, and Hilbert-Schmidt composition operators on $H^{2}$, Indiana Univ. Math. J. 125 (1973), 471-496. MR 48:4816

[Stn] C. S. Stanton, Riesz mass and growth problems for subharmonic functions, Thesis, Univ. of Wisconsin, Madison, 1982.

Department of Mathematics Education, Sung Kyun Kwan University, Jongro-Gu, SEOUl 110-745, Korea

E-mail address: jschoa@yurim.skku.ac.kr

Department of Mathematics, Korea Advanced Institute of Science and Technology, 373-1 Yusung-Gu Gusung-Dong, Taejun 305-701, Korea 